Research Article

\title{
Extension of Zhou's Method to Neutral Functional-Differential Equation with Proportional Delays
}

\author{
Sabir Widatalla ${ }^{1,2}$ \\ ${ }^{1}$ Department of Mathematics, Harbin Institute of Technology, Harbin 150001, China \\ ${ }^{2}$ Department of Mathematics, Sinnar University, Singa 107, Sudan
}

Correspondence should be addressed to Sabir Widatalla, sabirtag@yahoo.com

Received 30 January 2012; Accepted 19 February 2012

Academic Editors: C.-H. Lien and S. Prudhomme

Copyright (c) 2012 Sabir Widatalla. This is an open access article distributed under the Creative Commons Attribution License, which permits unrestricted use, distribution, and reproduction in any medium, provided the original work is properly cited.

The differential transform method (DTM) is a reliable method applied by providing new theorems to develop exact and approximate solutions of neutral functional-differential equation (NFDE) with proportional delays. The results obtained with the proposed methods are in good agreement with one obtained by other methods. The advantages of this technique are illustrated. It is easy to see that the DTM is very accurate and easy to implement in finding analytical solutions of wide classes of linear and nonlinear NFDEs.

\section{Introduction}

The neutral functional-differential equation (NFDE) is

$$
\left(u(t)+a(t) u\left(\sigma_{m}(t)\right)\right)^{(m)}=\beta u(t)+\sum_{k=0}^{m-1} b_{k}(t) u^{k}\left(\sigma_{k}(t)\right)+f(t), \quad t \geq 0,
$$

under the conditions

$$
\sum_{k=0}^{m-1} c_{i k} u^{(k)}(0)=\lambda_{i}, \quad i=0,1, \ldots, m-1,
$$

where $a, b_{k}, \sigma_{k}$ are analytical functions; $\beta, c_{i k}$ and $\lambda_{i} \in \mathcal{C}$. A classical case [1]

$$
\sigma_{k}(t)=t-\tau_{k}, \quad k=0,1, \ldots, m,
$$


where $\tau_{k}$ is positive. Another interesting case [2] is

$$
\sigma_{k}(t)=q_{k} t, \quad k=0,1, \ldots, m,
$$

where $0<q_{k}<1$. Both cases are playing an interesting role in many applications (see $[1,2]$ and references therein). In recent years, there has been a growing interest in the numerical treatment of NFDE, some of which are the Adams method [3], continuous Runge-Kutta methods [4], segmented Tau approximation [5], Homotopy perturbation method [6], one$\operatorname{leg} \theta$-methods $[7,8]$, and variational iteration method [9].

In this paper we consider the following neutral functional-differential equations with proportional delays.

Problem 1. Consider

$$
\begin{gathered}
u^{\prime}(t)=\beta u(t)-a(t) u^{\prime}\left(q_{\ell} t\right)+f\left(t, u(t), u\left(q_{i} t\right), u^{\prime}\left(q_{i} t\right)\right), \quad i=1,2, \ldots, \ell-1, \\
u(0)=u_{0} .
\end{gathered}
$$

Problem 2. Consider

$$
\begin{gathered}
u^{(m)}(t)=\beta u(t)-a(t) u^{(m)}\left(q_{\ell} t\right)+f\left(t, u(t), u\left(q_{i} t\right), u^{\prime}\left(q_{i} t\right), u^{\prime \prime}\left(q_{i} t\right), \ldots, u^{(m-1)}\left(q_{i} t\right)\right), \\
\sum_{k=0}^{m-1} c_{j k} u^{(k)}(0)=\lambda_{j}, \quad j=0,1,2, \ldots, m-1,
\end{gathered}
$$

where $a, f$ are analytical functions; $\beta, c_{j k}$ and $\lambda_{j}$ are real or complex constants; $0<q_{i}<1, i=$ $1,2, \ldots, \ell$.

The basic motivation of this work is to extend the differential transform method (DTM) by presenting and proving new theorems to create the exact or approximate solutions to a high degree of accuracy to the Problems 1 and 2. The DTM is a numerical-analytical technique that was first proposed by Zhou (1986) [10], who solved problems in electric circuit analysis. Since then, DTM was successfully applied for a large variety problems. For example, differential-difference equations [11], Volterra integral equation with separable kernels [12], MHD boundary-layer equations [13], linear and nonlinear systems of partial differential equations [14], and nonlinear oscillators with fractional nonlinearities [15]. To the best of our knowledge differential transform method has not be used by any researcher before to solve NFDE. By this method it is possible to obtain highly accurate results when compared with existing results from variational iteration method [9] and homotopy perturbation method [6]. 
Table 1: Fundamental operations of differential transformation.

\begin{tabular}{|c|c|}
\hline Original function & Transformed function \\
\hline$u(t)=f(t) \pm g(t)$ & $U(k)=F(k) \pm G(k)$ \\
\hline$u(t)=c f(t)$ & $U(k)=c F(k), c \in R$ \\
\hline$u(t)=f(t) g(t)$ & $U(k)=\sum_{\ell=0}^{k} F(\ell) G(k-\ell)$ \\
\hline$u(t)=f_{1}(t) f_{2}(t) \cdots f_{m-1}(t) f_{m}(t)$ & $\begin{aligned} U(k)= & \sum_{\ell_{m-1}=0}^{k} \sum_{\ell_{m-2}=0}^{\ell_{m-1}} \cdots \sum_{\ell_{2}=0}^{\ell_{3}} \times \sum_{\ell_{1}=0}^{\ell_{2}} F_{1}\left(\ell_{1}\right) \\
& \times F_{2}\left(\ell_{2}-\ell_{1}\right) \cdots F_{m-1}\left(\ell_{m-1}-\ell_{m-1}\right) F\left(k-\ell_{m-1}\right)\end{aligned}$ \\
\hline$u(t)=\left(d^{n} / d t^{n}\right) f(t)$ & $U(k)=((k+n) ! / k !) F_{m}(k)$ \\
\hline$u(t)=t^{m}$ & $U(k)=\delta(k-m)= \begin{cases}1, & k=m \\
0, & k \neq m\end{cases}$ \\
\hline
\end{tabular}

\section{Basic Idea of Differential Transform Method}

The differential transform of the $k$ th derivative of a function $u(t)$ is defined as follows:

$$
U(k)=\frac{1}{k !}\left[\frac{d^{k}}{d t^{k}} u(t)\right]_{t=t_{0}} .
$$

The original and transformed functions are denoted throughout this paper by small and capital letters, respectively. The differential inverse transform of $U(k)$ is defined as

$$
u(t)=\sum_{k=0}^{N} U(k)\left(t-t_{0}\right)^{k}, \quad \text { for } N \longrightarrow \infty
$$

And from (2.1) and (2.2):

$$
u(t)=\sum_{k=0}^{N} \frac{1}{k !}\left[\frac{d^{k}}{d t^{k}} u(t)\right]_{t=t_{0}}\left(t-t_{0}\right)^{k}, \quad \text { for } N \longrightarrow \infty,
$$

which is actually the Taylor series expansion of $u(t)$ about the point $t_{0}$.

The fundamental mathematical operations performed by one-dimensional differential transform can be obtained from (1.5) and (1.6) in Table 1 (also, see [10]).

\section{Main Results}

In the following theorems, we find the differential transformations of given functions. These results are very useful in our approach for solving NFDEs.

Theorem 3.1. Suppose that $U(k), F(k)$, and $G(k)$ are the differential transformations of the functions $u(t), f(t)$, respectively, and $g(t)$ and $0<q, q_{i}<1$, for $i=1,2, \ldots, m$ :

(I) if $u(t)=f(q t)$, then $U(k)=q^{k} F(k)$,

(II) if $u(t)=d^{n} f(q t) / d(q t)^{n}$, then $U(k)=q^{k}((k+n) ! / k !) F(k+n)$, 
(III) if $u(t)=g(t) d^{n} f(q t) / d(q t)^{n}$, then

$$
U(k)=\sum_{\ell=0}^{k} q^{k-\ell} \frac{(k-\ell+n)}{(k-\ell) !} G(\ell) F(k-\ell+n),
$$

(IV) if $u(t)=\left(d^{n} / d\left(q_{1} t\right)^{n}\right)\left[f\left(q_{1} t\right)\right]\left(d^{m} / d\left(q_{2} t\right)^{m}\right)\left[g\left(q_{2} t\right)\right]$, then

$$
U(k)=\sum_{\ell=0}^{k} q_{1}^{\ell} q_{2}^{k-\ell} \frac{(\ell+n) !(k-\ell+m) !}{(k-\ell) !(\ell) !} F(\ell+n) G(k-\ell+m),
$$

(V) if $u(t)=\left(d^{n_{1}} / d\left(q_{1} t\right)^{n_{1}}\right)\left[f_{1}\left(q_{1} t\right)\right]\left(d^{n_{2}} / d\left(q_{2} t\right)^{n_{2}}\right)\left[f_{2}\left(q_{2} t\right)\right] \cdots\left(d^{n_{m-1}} / d\left(q_{m-1} t\right)^{n_{m-1}}\right)$

$$
\left[f_{m-1}\left(q_{m-1} t\right)\left(d^{n_{m}} / d\left(q_{m} t\right)^{n_{m}}\right)\left[f_{m}\left(q_{m} t\right)\right]\right. \text {, then }
$$

$$
\begin{aligned}
U(k)= & \sum_{\ell_{m-1}=0}^{k} \sum_{\ell_{m-2}=0}^{\ell_{m-1}} \cdots \sum_{\ell_{2}=0}^{\ell_{3}} \sum_{\ell_{1}=0}^{\ell_{2}} q_{1}^{\ell_{1}} q_{2}^{\ell_{2}-\ell_{1}} \cdots q_{m-1}^{\ell_{m-1}-\ell_{m-2}} q_{m}^{\ell_{k}-\ell_{m-1}} \\
& \times \frac{\left(\ell_{1}+n_{1}\right) !}{\ell_{1} !} \frac{\left(\ell_{2}-\ell_{1}+n_{2}\right) !}{\left(\ell_{2}-\ell_{1}\right) !} \cdots \frac{\left(\ell_{m-1}-\ell_{m-2}+n_{m-1}\right) !}{\left(\ell_{m-1}-\ell_{m-2}\right) !} \frac{\left(k-\ell_{m-1}+n_{m}\right) !}{\left(k-\ell_{m-1}\right) !} \\
& \times F_{1}\left(\ell_{1}+n_{1}\right) F_{2}\left(\ell_{2}-\ell_{1}+n_{2}\right) \cdots F_{m-1}\left(\ell_{m-1}-\ell_{m-1}+n_{m-1}\right) F_{m}\left(k-\ell_{m-1}+n_{m}\right) .
\end{aligned}
$$

Proof. (I), (II) The proof follows immediately by substituting $u(t)$ into (2.1).

(III) By using the definition of DTM (2.1), we have

$$
\begin{aligned}
U(k) & =\frac{1}{k !}\left[\frac{d^{k}}{d t^{k}}\left(g(t) \frac{d^{n}}{d(q t)^{n}} f(q t)\right)\right]_{t=t_{0}} \\
& =\frac{1}{k !}\left[\sum_{\ell=0}^{k}\left(\begin{array}{l}
k \\
\ell
\end{array}\right) \frac{d^{\ell}}{d t^{\ell}} g(t) \frac{d^{k-\ell}}{d t^{k-\ell}}\left(\frac{d^{n}}{d(q t)^{n}} f(q t)\right)\right]_{t=t_{0}},
\end{aligned}
$$

and from (II), we have

$$
U(k-\ell)=\frac{1}{(k-\ell) !}\left[\frac{d^{k-\ell}}{d t^{k-\ell}} \frac{d^{n}}{d(q t)^{n}} f(q t)\right]_{t=t_{0}}=q^{k-\ell} \frac{(k-\ell+n) !}{(k-\ell) !} F(k-\ell+n) .
$$

By utilizing this value, we get

$$
U(k)=\sum_{\ell=0}^{k} q^{k-\ell} \frac{(k-\ell+n) !}{(k-\ell) !} G(\ell) F(k-\ell+n) .
$$


(IV) By using the definition of DTM (2.1), we have

$$
\begin{aligned}
U(k) & =\frac{1}{k !}\left[\frac{d^{k}}{d t^{k}}\left[\frac{d^{n}}{d\left(q_{1} t\right)^{n}}\left[f\left(q_{1} t\right)\right] \frac{d^{m}}{d\left(q_{2} t\right)^{m}}\left[f\left(q_{2} t\right)\right]\right]\right]_{t=t_{0}} \\
& =\frac{1}{k !}\left[\sum_{\ell=0}^{k}\left(\begin{array}{l}
k \\
\ell
\end{array}\right) \frac{d^{\ell}}{d t^{\ell}}\left[\frac{d^{n}}{d\left(q_{1} t\right)^{n}} f\left(q_{1} t\right)\right] \frac{d^{k-\ell}}{d t^{k-\ell}}\left[\frac{d^{m}}{d\left(q_{2 t}\right)^{m}} g\left(q_{2} t\right)\right]\right]_{t=t_{0}},
\end{aligned}
$$

then from (II), we have

$$
\begin{aligned}
U(\ell) & =\frac{1}{\ell !}\left[\frac{d^{\ell}}{d t^{\ell}} \frac{d^{n}}{d\left(q_{1} t\right)^{n}} f\left(q_{1} t\right)\right]=q_{1}^{\ell} \frac{(\ell+n) !}{\ell !} F(\ell+n), \\
U(k-\ell) & =\frac{1}{(k-\ell) !}\left[\frac{d^{k-\ell}}{d t^{k-\ell}} \frac{d^{m}}{d\left(q_{2} t\right)^{m}} g\left(q_{2} t\right)\right]=q_{2}^{k-\ell} \frac{(k-\ell+m) !}{(k-\ell) !} G(k-\ell+m) .
\end{aligned}
$$

By utilizing these values

$$
U(k)=\sum_{\ell=0}^{k} q_{1}^{\ell} q_{2}^{k-\ell} \frac{(\ell+n) !(k-\ell+m) !}{(k-\ell) !(\ell) !} F(\ell+n) G(k-\ell+m)
$$

(V) Let the differential transform of $d^{n_{i}} f_{i} / d\left(q_{i} t\right)^{n_{i}}$ at $t=t_{0}$ for $i=1,2, \ldots, m$ be $H_{i}(k)$, then by using operations of differential transformation given in Table 1, we have

$$
U(k)=\sum_{\ell_{m-1}=0}^{k} \sum_{\ell_{m-2}=0}^{\ell_{m-1}} \cdots \sum_{\ell_{2}=0}^{\ell_{3}} \sum_{\ell_{1}=0}^{\ell_{2}} H_{1}\left(\ell_{1}\right) H_{2}\left(\ell_{2}-\ell_{1}\right) \cdots H_{m-1}\left(\ell_{m-1}-\ell_{m-1}\right) H\left(k-\ell_{m-1}\right),
$$

and from (II), we have

$$
\begin{gathered}
H_{1}\left(\ell_{1}\right)=q_{1}^{\ell_{1}} \frac{\left(\ell_{1}+n_{1}\right) !}{\ell_{1} !} F_{1}\left(\ell_{1}+n_{1}\right) \\
H_{2}\left(\ell_{2}-\ell_{1}\right)=q_{2}^{\ell_{2}-\ell_{1}} \frac{\left(\ell_{2}-\ell_{1}+n_{2}\right) !}{\left(\ell_{2}-\ell_{1}\right) !} F_{2}\left(\ell_{2}-\ell_{1}+n_{1}\right) \\
\vdots \\
H_{m-1}\left(\ell_{m-1}-\ell_{m-2}\right)= \\
q_{m-1}^{\ell_{m-1}-\ell_{m-2}} \frac{\left(\ell_{m-1}-\ell_{m-2}+n_{m-1}\right) !}{\left(\ell_{m-1}-\ell_{m-21}\right) !} F_{m-1}\left(\ell_{m-1}-\ell_{m-2}+n_{m-1}\right) \\
H_{m}\left(k-\ell_{m-1}\right)= \\
q_{m}^{k-\ell_{m-1}} \frac{\left(k-\ell_{m-1}+n_{m}\right) !}{\left(k-\ell_{m-1}\right) !} F_{m}\left(k-\ell_{m-1}+n_{m}\right) .
\end{gathered}
$$

Substituting those values into (3.10), we obtain (3.3). 


\section{Illustrative Examples}

In this part, we will apply the DTM to solve NFDE with proportional delays.

The numerical solutions of Examples 4.3, 4.4, and 4.5 have been calculated by variational iteration method [9] and homotopy perturbation method [6], which did not yield the exact solutions. However, applying DTM gives the exact solutions of those examples, as we will show later.

Example 4.1 (see $[6,9]$ ). Consider the following first-order NFDE with proportional delay:

$$
\begin{gathered}
u^{\prime}(t)=-u(t)+\frac{1}{2} u\left(\frac{t}{2}\right)+\frac{1}{2} u^{\prime}\left(\frac{t}{2}\right), \quad 0<t<1, \\
u(0)=1 .
\end{gathered}
$$

Taking the differential transform of (4.1) as given in (2.1), we get

$$
(k+1) U(k+1)=-U(k)+\frac{1}{2}\left(\frac{1}{2}\right)^{k} U(k)+\frac{1}{2}\left(\frac{1}{2}\right)^{k}(k+1) U(k+1),
$$

which can be rewritten as follows:

$$
U(k+1)=\frac{-U(k)}{(k+1)}
$$

The differential transform of the initial condition of $u(t)$ at $t_{0}=0$, is $U(0)=1$, form (4.3) for $k=0,1, \ldots, 8$, we can get

$$
\begin{aligned}
& U(1)=-1, \quad U(2)=\frac{1}{2 !}, \quad U(3)=-\frac{1}{3 !}, \quad U(4)=\frac{1}{4 !}, \\
& U(5)=-\frac{1}{5 !}, \quad U(6)=\frac{1}{6 !}, \quad U(7)=-\frac{1}{7 !}, \quad U(8)=\frac{1}{8 !},
\end{aligned}
$$

substituting these values into (2.2), to get

$$
u(t)=1-t+\frac{t^{2}}{2 !}-\frac{t^{3}}{3 !}+\frac{t^{4}}{4 !}-\frac{t^{5}}{5 !}+\frac{t^{6}}{6 !}-\frac{t^{7}}{7 !}+\frac{t^{8}}{8 !}
$$

The closed form of the above solution, when $N \rightarrow \infty$ is $u(t)=e^{-t}$, which is the exact solution. In Table 2 the absolute errors of DTM for $N=7,8$, VIM [9] with eight terms and HPM [6] with eight terms (Table 3 ) are compared.

Example 4.2 (see $[6,9]$ ). Consider the first-order NFDE with proportional delay

$$
\begin{gathered}
u^{\prime}(t)=-u(t)+0.1 u(0.8 t)+0.5 u^{\prime}(0.8 t)+(0.32 t-0.5) e^{-0.8 t}+e^{-t}, \quad t \geq 0, \\
u(0)=0 .
\end{gathered}
$$


Table 2: Comparison of the absolute errors for Example 4.1.

\begin{tabular}{lcccc}
\hline$t$ & VIM [9] & HPM [6] & \multicolumn{2}{c}{ DTM } \\
& $n=8$ & $n=8$ & $n=7$ & $n=8$ \\
\hline 0.1 & $3.72 E-4$ & $3.36 E-4$ & $2.45 E-13$ & $2.89 E-15$ \\
0.2 & $7.08 E-4$ & $5.80 E-4$ & $6.21 E-11$ & $1.38 E-12$ \\
0.3 & $1.01 E-3$ & $7.50 E-4$ & $1.57 E-9$ & $5.27 E-11$ \\
0.4 & $1.29 E-3$ & $8.64 E-4$ & $1.56 E-8$ & $6.95 E-10$ \\
0.5 & $1.54 E-3$ & $9.33 E-4$ & $9.18 E-8$ & $5.12 E-9$ \\
0.6 & $1.76 E-3$ & $9.68 E-4$ & $3.90 E-7$ & $2.62 E-8$ \\
0.7 & $1.97 E-3$ & $9.78 E-4$ & $1.32 E-6$ & $1.04 E-7$ \\
0.8 & $2.15 E-3$ & $9.68 E-4$ & $3.82 E-6$ & $3.42 E-7$ \\
0.9 & $2.32 E-3$ & $9.44 E-4$ & $9.70 E-6$ & $9.79 E-7$ \\
1.0 & $2.47 E-3$ & $9.10 E-4$ & $2.23 E-5$ & $2.50 E-6$ \\
\hline
\end{tabular}

Table 3: Comparison of the absolute errors for Example 4.2.

\begin{tabular}{lcccc}
\hline$t$ & VIM [9] & HPM [6] & \multicolumn{2}{c}{ DTM } \\
& $n=8$ & $n=8$ & $n=7$ & $n=8$ \\
\hline 0.1 & $1.30 E-3$ & $1.06 E-3$ & $8.20 E-9$ & $1.37 E-10$ \\
0.2 & $2.14 E-3$ & $1.35 E-3$ & $5.16 E-7$ & $1.73 E-8$ \\
0.3 & $2.63 E-3$ & $1.18 E-3$ & $5.78 E-6$ & $2.91 E-7$ \\
0.4 & $2.84 E-3$ & $7.61 E-4$ & $3.20 E-5$ & $2.15 E-6$ \\
0.5 & $2.83 E-3$ & $2.32 E-4$ & $1.20 E-4$ & $1.01 E-5$ \\
0.6 & $2.67 E-3$ & $2.98 E-4$ & $3.53 E-4$ & $3.58 E-5$ \\
0.7 & $2.39 E-3$ & $7.64 E-4$ & $8.77 E-4$ & $1.04 E-4$ \\
0.8 & $2.04 E-3$ & $1.12 E-3$ & $1.92 E-3$ & $2.61 E-4$ \\
0.9 & $1.64 E-3$ & $1.37 E-3$ & $3.84 E-3$ & $5.88 E-4$ \\
1.0 & $1.22 E-3$ & $1.50 E-3$ & $7.12 E-3$ & $1.21 E-3$ \\
\hline
\end{tabular}

Let The differential transforms of $t e^{-0.8 t}, e^{-0.8 t}$, and $e^{-t}$ at $t_{0}=0$ be $\delta_{1}(k), \delta_{2}(k)$, and $\delta_{3}(k)$, respectively:

$$
\begin{aligned}
& \delta_{1}(k)= \begin{cases}0, & k=0, \\
\frac{(-1)^{k-1}(0.8)^{k-1}}{k-1} !, & k \neq 0,\end{cases} \\
& \delta_{2}(k)=\frac{(-1)^{k}(0.8)^{k}}{k !}, \\
& \delta_{3}(k)=\frac{(-1)^{k}}{k !} .
\end{aligned}
$$


We can obtain the differential transform of (4.6) as

$$
U(k+1)=\frac{-U(k)\left(1-0.1(0.8)^{k}\right)+0.32 \delta_{1}(k)-0.5 \delta_{2}(k)+\delta_{3}(k)}{(k+1)\left(1-0.5(0.8)^{k}\right)} .
$$

At $t_{0}=0$, the initial condition transformed to $U(0)=0$, so from (4.8), we have

$$
\begin{gathered}
U(1)=1, \quad U(2)=-1, \quad U(3)=\frac{1}{2}, \quad U(4)=-\frac{1}{6}, \\
U(5)=\frac{1}{24}, \quad U(6)=-\frac{1}{120}, \quad U(7)=\frac{1}{720}, \quad U(8)=-\frac{1}{5040} .
\end{gathered}
$$

Substituting these values into(2.2), we get

$$
u(t)=t-t^{2}+\frac{1}{2} t^{3}-\frac{1}{6} t^{4}+\frac{1}{24} t^{5}-\frac{1}{120} t^{6}+\frac{1}{720} t^{7}-\frac{1}{5040} t^{8}
$$

The closed form of the above solution, when $N \rightarrow \infty$, is $u(t)=t e^{-t}$, which is the exact solution. In Table 2 we compare the absolute errors of DTM for $N=7,8$, VIM [9] with eight terms, and HPM [6] with eight terms.

Example 4.3 (see[6,9]). Consider the following second-order NFDE with proportional delay:

$$
\begin{gathered}
u^{\prime \prime}(t)=u^{\prime}\left(\frac{t}{2}\right)-\frac{1}{2} t u^{\prime \prime}\left(\frac{t}{2}\right)+2, \quad 0<t<1 \\
u(0)=1, \quad u^{\prime}(0)=0 .
\end{gathered}
$$

The differential transform for (4.11) is found as

$$
\begin{aligned}
& (k+1)(k+2) U(k+2) \\
& =\frac{1}{2^{k}}(k+1) U(k+1)-\frac{1}{2} \sum_{\ell=0}^{k} \frac{1}{2^{k-\ell}} \frac{(k-\ell+2) !}{(k-\ell) !} \delta(\ell-1) U(k-\ell+2)+2 \delta(k),
\end{aligned}
$$

form the initial condition we can get $U(0)=1$ and $U(1)=0$. Form (4.12), we get

$$
U(k)= \begin{cases}1, & k=2 \\ 0, & k>2\end{cases}
$$

Then, by using (2.2), $u(t)=1+t^{2}$, which is the exact solution. 
Example 4.4 (see $[6,9]$ ). Consider the second-order NFDE with proportional delay:

$$
\begin{gathered}
u^{\prime \prime}(t)=\frac{3}{4} u(t)+u\left(\frac{t}{2}\right)+u^{\prime}\left(\frac{t}{2}\right)+\frac{1}{2} u^{\prime \prime}\left(\frac{t}{2}\right)-t^{2}-t+1, \quad 0<t<1, \\
u(0)=u^{\prime}(0)=0 .
\end{gathered}
$$

The differential transform for (4.14) at $t_{0}=0$ is given by

$$
\begin{aligned}
(k+1)(k+2) U(k+2)= & \frac{3}{4} U(k)+\frac{1}{2^{k}} U(k)+\frac{1}{2^{k}}(k+1) U(k+1)+\frac{1}{2} \frac{1}{2^{k}}(k+1) \\
& \times(k+2) U(k+2)-\delta(k-2)-\delta(k-1)+\delta(k),
\end{aligned}
$$

and can be rewritten as

$$
U(k+2)=\frac{\left(3 / 4+1 / 2^{k}\right) U(k)+\left(1 / 2^{k}\right)(k+1) U(k+1)-\delta(k-2)-\delta(k-1)+\delta(k)}{(k+1)(k+2)\left(1-1 / 2^{k+1}\right)} .
$$

Form the initial condition we can get $U(0)=U(1)=0$, and form (4.16), we get

$$
U(k)= \begin{cases}1, & k=2 \\ 0, & k>2\end{cases}
$$

Then, by using (2.2), $u(t)=t^{2}$, which is the exact solution.

Example 4.5 (see $[6,9]$ ). Consider the following third-order NFDE with proportional delays:

$$
\begin{gathered}
u^{\prime \prime \prime}(t)=u(t)+u^{\prime}\left(\frac{t}{2}\right)+u^{\prime \prime}\left(\frac{t}{3}\right)+\frac{1}{2} u^{\prime \prime \prime}\left(\frac{t}{4}\right)-t^{4}-\frac{t^{3}}{2}-\frac{4 t^{2}}{3}+21 t \\
u(0)=0, \quad u^{\prime}(0)=0, \quad u^{\prime \prime}(0)=0 .
\end{gathered}
$$

The differential transform of (4.18) can be written as

$$
\begin{gathered}
U(k)+\frac{1}{2^{k}}(k+1) U(k+1)+\frac{1}{3^{k}}(k+1)(k+2) U(k+2) \\
U(k+3)=\frac{-\delta(k-4)-(1 / 2) \delta(k-3)-(4 / 3) \delta(k-2)+21 \delta(k-1)}{(k+1)(k+2)(k+3)\left(1-(1 / 2)\left(1 / 4^{k}\right)\right)} .
\end{gathered}
$$
get

Form the initial condition we can get $U(0)=U(1)=U(2)=0$, so from the (4.19), we

$$
U(k)= \begin{cases}0, & k \neq 4 \\ 1, & k=4\end{cases}
$$

Substituting (4.20) in (2.2) gives $u(t)=t^{4}$, which is the exact solution. 


\section{Conclusion}

In this study, we extended DTM to the solution of NFDE with proportional delays. New theorems are presented with their proofs. All examples results show that the DTM is more effective than VIM and HPM for solving NFDE with proportional delays. We believe that the ease of implementation and efficiency of the DTM gives it much wider applicability.

\section{References}

[1] O. Arino and O. Sidki, "An abstract neutral functional-differential equation arising from a cell population model," Journal of Mathematical Analysis and Applications, vol. 235, no. 2, pp. 435-453, 1999.

[2] A. Bellen and M. Zennaro, Numerical Methods for Delay Differential Equations, Numerical Mathematics and Scientific Computation, The Clarendon Press Oxford University Press, New York, NY, USA, 2003.

[3] Z. Jackiewicz and E. Lo, "Numerical solution of neutral functional differential equations by Adams methods in divided difference form," Journal of Computational and Applied Mathematics, vol. 189, no. 1-2, pp. 592-605, 2006.

[4] W. Wang, Y. Zhang, and S. Li, "Stability of continuous Runge-Kutta-type methods for nonlinear neutral delay-differential equations," Applied Mathematical Modelling, vol. 33, no. 8, pp. 3319-3329, 2009.

[5] L. F. Cordero and R. Escalante, "Segmented tau approximation for test neutral functional differential equations," Applied Mathematics and Computation, vol. 187, no. 2, pp. 725-740, 2007.

[6] J. Biazar and B. Ghanbari, "The homotopy perturbation method for solving neutral functionaldifferential equations with proportional delays," Journal of King Saud University - Science, vol. 24, pp. 33-37, 2010.

[7] W.-S. Wang and S.-F. Li, "On the one-leg $\theta$-methods for solving nonlinear neutral functional differential equations," Applied Mathematics and Computation, vol. 193, no. 1, pp. 285-301, 2007.

[8] W. Wang, T. Qin, and S. Li, "Stability of one-leg $\theta$-methods for nonlinear neutral differential equations with proportional delay," Applied Mathematics and Computation, vol. 213, no. 1, pp. 177-183, 2009.

[9] X. Chen and L. Wang, "The variational iteration method for solving a neutral functional-differential equation with proportional delays," Computers $\mathcal{E}$ Mathematics with Applications, vol. 59, no. 8, pp. 2696-2702, 2010.

[10] J. K. Zhou, Differential Transformation and its Application for Electrical Circuit, Huazhong University Press, Wuhan, China, 1986.

[11] A. Arikoglu and I. Ozkol, "Solution of differential-difference equations by using differential transform method," Applied Mathematics and Computation, vol. 181, no. 1, pp. 153-162, 2006.

[12] Z. M. Odibat, "Differential transform method for solving Volterra integral equation with separable kernels," Mathematical and Computer Modelling, vol. 48, no. 7-8, pp. 1144-1149, 2008.

[13] M. M. Rashidi, "The modified differential transform method for solving MHD boundary-layer equations," Computer Physics Communications, vol. 180, no. 11, pp. 2210-2217, 2009.

[14] A. S. V. Ravi Kanth and K. Aruna, "Differential transform method for solving linear and non-linear systems of partial differential equations," Physics Letters A, vol. 372, no. 46, pp. 6896-6898, 2008.

[15] A. E. Ebaid, "A reliable aftertreatment for improving the differential transformation method and its application to nonlinear oscillators with fractional nonlinearities," Communications in Nonlinear Science and Numerical Simulation, vol. 16, no. 1, pp. 528-536, 2011. 


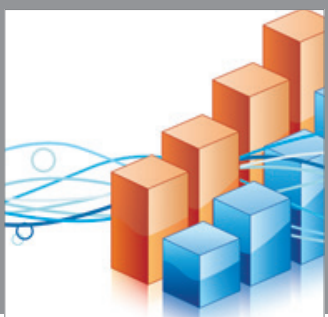

Advances in

Operations Research

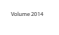

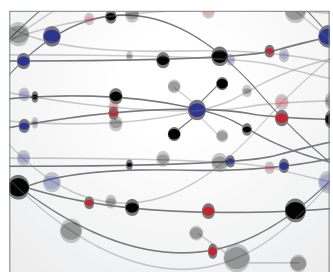

\section{The Scientific} World Journal
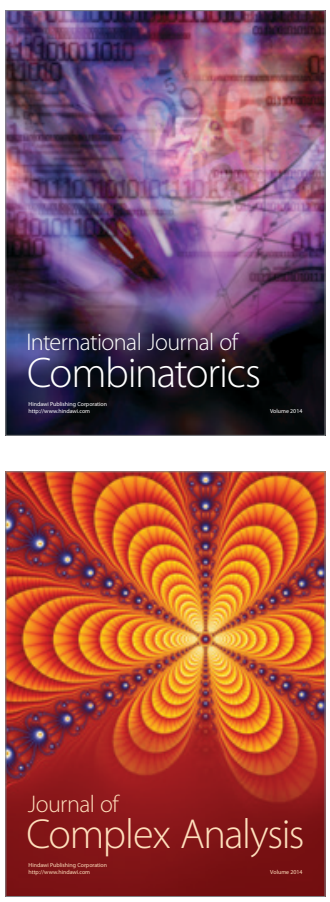

International Journal of

Mathematics and

Mathematical

Sciences
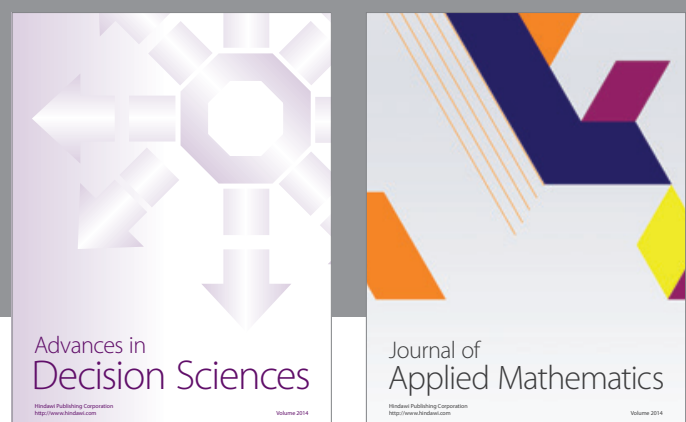

Journal of

Applied Mathematics
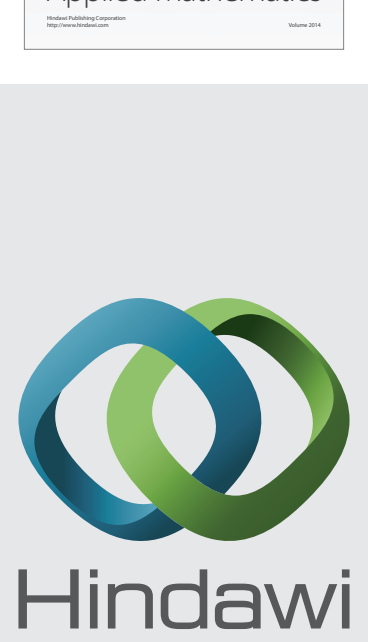

Submit your manuscripts at http://www.hindawi.com
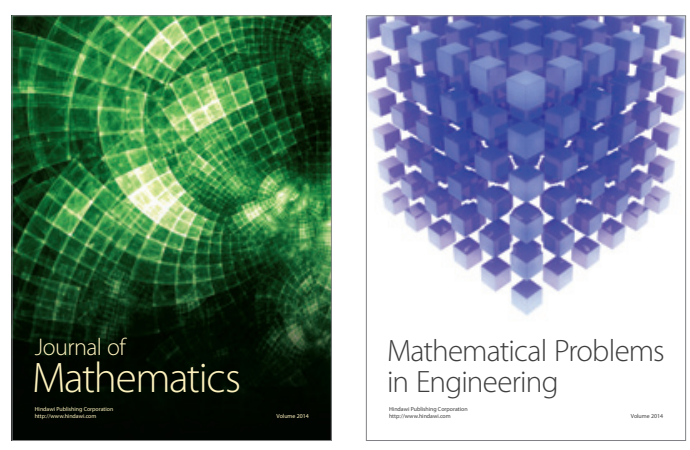

Mathematical Problems in Engineering
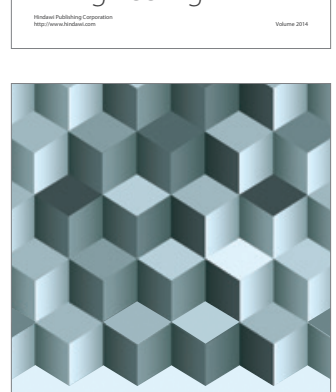

Journal of

Function Spaces
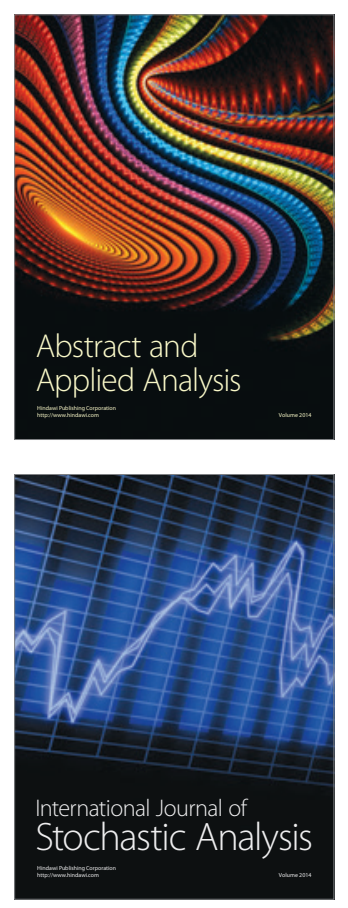

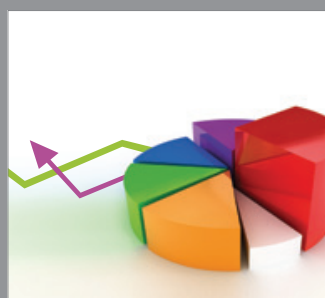

ournal of

Probability and Statistics

Promensencen
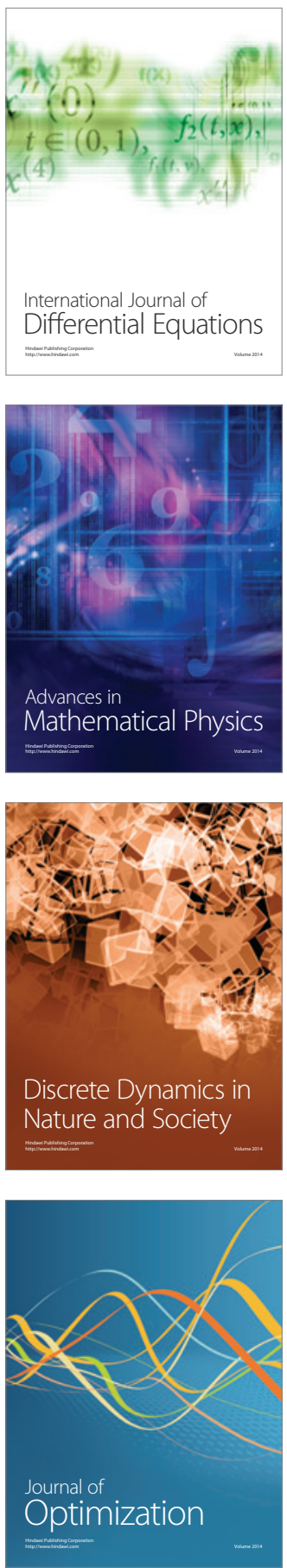